VoL. 67 (2003) [249-256]

\title{
IMPROVEMENT ON THE BOUNDS OF PERMUTATION GROUPS WITH BOUNDED MOVEMENT
}

\author{
Mehdi Alaeiyan
}

Let $G$ be a permutation group on a set $\Omega$ with no fixed points in $\Omega$ and let $m$ be a positive integer. Then we define the movement of $G$ as, $m:=\operatorname{move}(G)$ $:=\sup _{\Gamma}\left\{\left|\Gamma^{g} \backslash \Gamma\right| \mid g \in G\right\}$. Let $p$ be a prime, $p \geqslant 5$. If $G$ is not a 2-group and $p$ is the least odd prime dividing $|G|$, then we show that $n:=|\Omega| \leqslant 4 m-p+3$.

Moreover, if we suppose that the permutation group induced by $G$ on each orbit is not a 2-group then we improve the last bound of $n$ and for an infinite family of groups the bound is attained.

\section{INTRODUCTION}

Let $G$ be a permutation group on a set $\Omega$ with no fixed points in $\Omega$ and let $m$ be a positive integer. If for each subset $\Gamma$ of $\Omega$ the size $\left|\Gamma^{g} \backslash \Gamma\right|$ is bounded, for $g \in G$, we define the movement of $\Gamma$ as move $(\Gamma)=\max _{g \in G}\left|\Gamma^{g} \backslash \Gamma\right|$. If move $(\Gamma) \leqslant m$ for all $\Gamma \subseteq \Omega$, then $G$ is said to have bounded movement and the movement of $G$ is defined as the maximum of move $(\Gamma)$ over all subsets $\Gamma$, that is,

$$
m:=\operatorname{move}(G):=\sup \left\{\left|\Gamma^{g} \backslash \Gamma\right| \mid \Gamma \subseteq \Omega, g \in G\right\}
$$

This notion was introduced in [4]. By [4, Theorem 1], if $G$ has movement $m$, then $\Omega$ is finite. Moreover both the number of $G$-orbits in $\Omega$ and the length of each $G$-orbit are bounded above by linear functions of $m$. In particular, it was proved that each $G$-orbit has length at most $3 m$ and $n:=|\Omega| \leqslant 5 m-2$. In [1] it was shown that $n=5 m-2$ if and only if $n=3$ and $G$ is transitive. But in [3], this bound was refined further and it was shown that $n \leqslant(9 m-3) / 2$. Moreover, if $n=(9 m-3) / 2$ then either $n=3$ and $G=S_{3}$ or $G$ is an elementary Abelian 3-group and all its orbits have length 3. Now suppose that $G$ is not a 2-group, and let $p(\geqslant 5)$ be the least odd prime dividing the $|G|$. Then by $[4$, Lemma 2,2$], n \leqslant(9 m-3) / 2$. In this paper we aim to improve the bound for any group with above conditions as follows:

THEOREM 1.1. Let $p$ be a prime, $p \geqslant 5$, let $m$ be a positive integer, and let $G$ be a permutation group on a set $\Omega$ with movement $m$ such that $G$ has no fixed points in $\Omega$. If $G$ is not a 2-group and $p$ is the least odd prime dividing $|G|$, then $n:=|\Omega| \leqslant 4 m-p+3$.

Received 24th July, 2002

Copyright Clearance Centre, Inc. Serial-fee code: 0004-9727/03 \$A2.00+0.00. 
We do not know whether the bound in Theorem 1.1 is attained. But according to hypothesis in Theorem 1.1, and with the assumption that $G$ is not a 2-group on each orbit, then we have the following result such that the bound is attained for an infinite family of groups. We denote by $P \ltimes K$ a semi-direct product $K . P$ with normal subgroup $K$.

THEOREM 1.2. Let $G$ be a permutation group on a set $\Omega$ with movement $m$ such that $G$ has no fixed points in $\Omega$. If $G$ is not a 2-group on any orbit, and $p \geqslant 5$ is the least odd prime dividing $|G|$, then the following hold:

1. $n:=|\Omega| \leqslant(p / p-1)(\lfloor(2 m p / p-1)\rfloor-1)$ (for $x \in \mathbb{R},\lfloor x\rfloor$ denotes the integer part of $x$ );

2. moreover, if $|\Omega|=(p / p-1)(\lfloor(2 m p / p-1)\rfloor-1)$ then either $n=p, m$ $=(p-1) / 2$, and $G: \leqslant Z_{2^{a} \mathrm{o}} \ltimes Z_{p}$ where $a_{0}=\max \left\{a \mid 2^{a}\right.$ divides $\left.p-1\right\}$, that is, $2^{\alpha_{0}}$ is the 2-part of $(p-1)$, or for some positive integer $d, G \cong Z_{p}^{d}$, $m=p^{d-1}(p-1) / 2$ and all its orbits have length $p$.

\section{EXAMPles AND PRELiminaries}

Let $1 \neq g \in G$ and suppose that $g$ in its disjoint cycle representation has $t$ nontrivial cycles of lengths $l_{1}, \ldots, l_{t}$ say. We might represent $g$ as

$$
g=\left(a_{1} a_{2} \ldots a_{l_{1}}\right)\left(b_{1} b_{2} \ldots b_{l_{2}}\right) \ldots\left(z_{1} z_{2} \ldots z_{l_{t}}\right)
$$

Let $\Gamma(g)$ denote a subset of $\Omega$ consisting of $\left\lfloor l_{i} / 2\right\rfloor$ points from the $i^{\text {th }}$ cycle, for each $i$, chosen in such a way that $\Gamma(g)^{g} \cap \Gamma(g)=\emptyset$. For example, we could choose $\Gamma(g)=\left\{a_{2}, a_{4}, \ldots, b_{2}, b_{4}, \ldots, z_{2}, z_{4}, \ldots\right\}$. Note that $\Gamma(g)$ is not uniquely determined as it depends on the way each cycle is written. For any set $\Gamma(g)$ of this kind we say that $\Gamma(g)$ consists of every second point of every cycle of $g$. From the definition of $\Gamma(g)$ we see that

$$
\left|\Gamma(g)^{g} \backslash \Gamma(g)\right|=|\Gamma(g)|=\sum_{i=1}^{t}\left\lfloor l_{i} / 2\right\rfloor .
$$

The next lemma shows that this quantity is an upper bound for $\left|\Gamma^{g} \backslash \Gamma\right|$ for an arbitrary subset $\Gamma$ of $\Omega$.

LEMMA 2.1 ([2, Lemma 2.1].) Let $G$ be a permutation group on a set $\Omega$ and suppose that $\Gamma \subseteq \Omega$. Then for each $g \in G,\left|\Gamma^{g} \backslash \Gamma\right| \leqslant \sum_{i=1}^{t}\left\lfloor l_{i} / 2\right\rfloor$ where $l_{i}$ is the length of the $i^{\text {th }}$ cycle of $g$ and $t$ is the number of nontrivial cycles of $g$ in its disjoint cycle representation. This upper bound is attained for $\Gamma=\Gamma(g)$ defined above.

Now we shall show that there certainly is an infinite family of groups for which equality in Theorem 1.2(1) holds, for any prime $p$. 
EXAMPLE 2.2. Let $d$ be a positive integer, let $G:=Z_{p}^{d}$, let $t:=\left(p^{d}-1\right) /(p-1)$, and let $H_{1}, \ldots, H_{t}$ be an enumeration of the subgroups of index $p$ in $G$. Define $\Omega_{i}$ to be the coset space of $H_{i}$ in $G$ and $\Omega=\Omega_{1} \cup \ldots \cup \Omega_{t}$. If $g \in G-\{1\}$, then $g$ lies in $\left(p^{d-1}-1\right) /(p-1)$ of the groups $H_{i}$ and therefore acts on $\Omega$ as a permutation with $(p / p-1)\left(p^{d-1}-1\right)$ fixed points and $p^{d-1}$ orbits of length $p$. It follows from Lemma 2.1 that $\operatorname{move}(G)=m=p^{d-1}(p-1) / 2$. Thus $\lfloor(2 m p / p-1)\rfloor=p^{d}$ and

$$
n=|\Omega|=p \cdot t=\frac{p}{p-1}\left(p^{d}-1\right)=\frac{p}{p-1}\left(\left\lfloor\frac{2 m p}{p-1}\right\rfloor-1\right) .
$$

Now we have the following lemma which is a classification of all transitive permutation groups $G$ of degree $p$ where $p$ is the least odd prime dividing $|G|$.

LEMMA 2.3. Let $G$ be a transitive permutation group on a set $\Omega$ of size $p$, where $p \geqslant 5$ is the least odd prime dividing $|G|$. Then $G=Z_{2^{a}} \ltimes Z_{p}$, where $a \geqslant 0$, and $2^{a} \mid(p-1)$.

Proof: Let $G$ be a transitive permutation group on a set $\Omega$ of size $p$. Then $G$ is isomorphic to a transitive subgroup of $S_{p}$ and so $p$ is the largest prime divisor of $|G|$. Since $p$ is also the least odd prime dividing $|G|$, we have $|G|=p .2^{a}$ for some $a \geqslant 0$. By Burnside's "pq theorem" (see [6, Theorem 2, 10, 17]) $G$ is soluble, and hence by a theorem of Galois [6, Theorem 3.6.1], $G$ is isomorphic to a subgroup of the group $A G L(1, p)$ of affine transformations of a finite field consisting of $p$ elements. Thus $G=Z_{2^{\alpha}} \times Z_{p}$ as asserted.

COROLlaRY 2.4. Let $G$ be a permutation group on a set $\Omega$, and suppose that $\Delta$ is a $G$-orbit of length $p$ in $\Omega$ where $p$ is the least odd prime dividing $|G|$. Then the induced permutation group $G^{\Delta}$ is $G=Z_{2^{a}} \ltimes Z_{p}$ where $0 \leqslant a \leqslant a_{0}$, and $2^{a_{0}}$ is the 2-part of $(p-1)$.

\section{The MAXIMUM DEgREe OF BOUNDED MOVEMENT GROUPS}

Suppose that $G \leqslant S y m(\Omega)$ and that $G$ is not a 2-group and $\operatorname{move}(G)=m$, and such that $p \geqslant 5$ is the least odd prime dividing the $|G|$. In this section we find an upper bound for $|\Omega|$ that is a linear function of $m$.

To prove the main theorems, we introduce the following notation.

$r_{p}:=$ number of $G$-orbits of length $p$ on which $G$ acts as $Z_{p}$;

$r_{p}^{\prime}(a):=$ number of $G$-orbits of length $p$ on which $G$ acts as $G=Z_{2^{a}} \ltimes Z_{p}$ with $1 \leqslant a \leqslant a_{0} ;$ and set $r_{p}^{\prime}:=\sum_{a=1}^{a_{0}} r_{p}^{\prime}(a)$;

$\Phi:=$ union of $G$-orbits of lengths $2^{b}$, where $1 \leqslant b \leqslant \log _{2} p$; and $u$ is the number of orbits in $\Phi$.

$s:=$ number of $G$-orbits of length $>p$. 
The orbits are labelled accordingly. Thus $\Omega_{1}, \ldots, \Omega_{r_{p}}$ are those of length $p$ on which $G$ acts as $Z_{p} ; \Omega_{r_{p}+1}, \ldots, \Omega_{r_{p}+r_{p}^{\prime}}$ are those of length $p$ on which $G$ acts as $G=Z_{2^{a}} \ltimes Z_{p}$ for some $a \geqslant 1 ; \Omega_{r_{p}+r_{p}^{\prime}+1}, \ldots, \Omega_{r_{p}+r_{p}^{\prime}+u}$ are those of length $2^{b}$ where $1 \leqslant b \leqslant \log _{2} p$; et cetera. Define $t:=r_{p}+r_{p}^{\prime}+u+s, t_{1}:=r_{p}+r_{p}^{\prime}+u$, and $t_{0}=r_{p}+r_{p}^{\prime}$. So $t$ is the total number of $G$-orbits.

For $1 \leqslant i \leqslant t_{0}$ define $K_{i}$ to be the kernel of the action of $G$ on $\Omega_{i}$ and for $g \in G$ define $k(g)$ to be the number of $i$ in that range for which $g$ is not in $K_{i}$. For $g \in G$ and a $G$-invariant set $\Delta$ we denote by $\operatorname{fix}_{\Delta}(g)=\left\{\alpha \in \Delta \mid \alpha^{g}=\alpha\right\}$ and $\operatorname{supp}_{\Delta}(g)=\{\alpha \in \Delta \mid$ $\left.\alpha^{g} \neq \alpha\right\}$ the set of fixed points of $g$ in $\Delta$ and the support of $g$ in $\Delta$, respectively (so that $\mid$ fix $\left._{\Delta}(g)|+| \operatorname{supp}_{\Delta}(g)|=| \Delta \mid\right)$, and define odd $\operatorname{od}_{\Delta}(g):=$ the number non-trivial cycles of $g$ in $\Delta$ that have odd length.

LEMMA 3.1. With the above notation, let $\Delta:=\bigcup_{i=t_{1}+1}^{t} \Omega_{i}$ and let $g \in G$. Then

$$
\frac{p-1}{2} k(g)+\frac{1}{2}\left|\operatorname{supp}_{\Phi}(g)\right|+\frac{1}{2}\left(\left|\operatorname{supp}_{\Delta}(g)\right|-\operatorname{odd}_{\Delta}(g)\right) \leqslant m \text {. }
$$

Proof: For each $i$ such that $1 \leqslant i \leqslant t_{0}$ and $g$ is not in $K_{i}$, since $\left|\Omega_{i}\right|=p$ then $g^{\Omega_{i}}$ is a $p$-cycle or a 2-element with one fixed point and we may choose a subset $\Gamma_{i}$ of $(p-1) / 2$ points of $\Omega_{i}$ such that $\Gamma_{i}^{g} \cap \Gamma_{i}=\emptyset$. Let $\Gamma_{0}$ be the set of chosen points from all the $\Gamma_{i}$ for $1 \leqslant i \leqslant t_{0}$, and so by definition $\Gamma_{0}^{g} \cap \Gamma_{0}=\emptyset$.

For each of the non-trivial cycles $\left(b_{1} \ldots b_{2 l}\right)$ and $\left(a_{1} a_{2} \ldots a_{k}\right)$ of $g$ in $\Phi$ and $\Delta$ respectively, adjoin the points $b_{1}, b_{3}, \ldots, b_{2 l-1}$ and also $a_{1}, a_{3}, \ldots, a_{k^{\prime}}$ to $\Gamma_{0}$, where $k^{\prime}$ is odd and $k-2 \leqslant k^{\prime}$ $\leqslant k-1$. Let $\Gamma$ be the resulting set. It has been constructed so that $\Gamma^{g} \cap \Gamma=\emptyset$. Therefore $|\Gamma| \leqslant m$. Since

$$
|\Gamma|=\frac{p-1}{2} k(g)+\frac{1}{2}\left|\operatorname{supp}_{\Phi}(g)\right|+\frac{1}{2}\left(\left|\operatorname{supp}_{\Delta}(g)\right|-\operatorname{odd}_{\Delta}(g)\right),
$$

we have the stated inequality.

To prove Theorem 1.1 we first prove the following lemma.

\section{LEMMA 3.2 :}

$$
\left(\sum_{a=0}^{a_{0}} \frac{p-1}{2} \cdot\left(1-\frac{1}{2^{a} p}\right) r_{p}^{\prime}(a)+\frac{|\Phi|-u}{2}+\frac{p-1}{2 p}(|\Delta|-s)\right)<m,
$$

where for $a=0$ the number $r_{p}^{\prime}(a)$ is $r_{p}$.

Proof: Suppose that $1 \leqslant i \leqslant t_{0}$. Then the group induced by $G$ on $\Omega_{i}$ is $G=Z_{2^{a}} \ltimes Z_{p}$ for some $a \geqslant 0$, such that $2^{a} \mid(p-1)$, and since $\left|G: K_{i}\right|=2^{a} p$, there are

$$
|G|-\left|K_{i}\right|=\left(2^{a} p-1\right)\left|K_{i}\right|
$$

elements $g$ which act nontrivially on $\Omega_{i}$. It follows that

$$
\sum_{g \in G} \frac{p-1}{2} k(g)=\frac{p-1}{2} \sum_{a=0}^{a_{0}}\left(\frac{2^{a} p-1}{2^{a} p}|G|\right) r_{p}^{\prime}(a)
$$


where $r_{p}^{\prime}(0):=r_{p}$

For $t_{0}+1 \leqslant i \leqslant t_{1}$, the group induced by $G$ on $\Omega_{i}$ is a 2-group. The union of these sets $\Omega_{i}$ is $\Phi$, and since by Burnside's Lemma [5, Theorem 3.26] the average number of fixed points of elements of $G$ in $\Phi$ is the number $u$ of G-orbits in $\Phi$, we have

$$
\sum_{g \in G} \frac{1}{2}\left|\operatorname{supp}_{\Phi}(g)\right|=\frac{1}{2} \sum_{g \in G}\left(|\Phi|-\left|\operatorname{fix}_{\Phi}(g)\right|\right)=\frac{1}{2}|\Phi| \cdot|G|-\frac{|G|}{2} \cdot u
$$

Similarly,

$$
\sum_{g \in G} \frac{1}{2}\left|\operatorname{supp}_{\Delta}(g)\right|=\frac{1}{2}|\Delta| \cdot|G|-\frac{s|G|}{2}
$$

and since $\operatorname{odd}_{\Delta}(g) \leqslant(1 / p)\left|\operatorname{supp}_{\Delta}(g)\right|$, we have

$$
\sum_{g \in G} \frac{1}{2}\left(\left|\operatorname{supp}_{\Delta}(g)\right|-\operatorname{odd}_{\Delta}(g)\right) \geqslant \frac{p-1}{2 p}(|\Delta| .|G|-s|G|) .
$$

Thus adding the inequality of lemma 3.1 over all $g \in G$, we obtain

$$
m|G|>|G|\left(\sum_{a=0}^{a_{0}} \frac{p-1}{2} \cdot\left(1-\frac{1}{2^{a} p}\right) r_{p}^{\prime}(a)+\frac{|\Phi|-u}{2}+\frac{p-1}{2 p}(|\Delta|-s)\right),
$$

where the strict inequality recognises the fact the inequality of Lemma 3.1 is strict for the identity element of $|G|$. This completes the proof of Lemma 3.2.

Now

$$
n=\left(\sum_{a=0}^{a_{0}} r_{p}^{\prime}(a)\right) p+|\Phi|+|\Delta| .
$$

Also we have $|\Phi| \geqslant 2 u$, and so

$$
\frac{|\Phi|-u}{2} \geqslant \frac{|\Phi|}{4}
$$

Thus the inequality in Lemma 3.2 implies that

$$
\begin{aligned}
m & >\frac{n}{4}+\sum_{a=0}^{a_{0}} r_{p}^{\prime}(a)\left(\frac{p-1}{2}-\frac{p-1}{2^{a+1} p}-\frac{p}{4}\right)+|\Delta|\left(\frac{p-1}{2 p}-\frac{1}{4}\right)-\frac{p-1}{2 p} s \\
& =\frac{n}{4}+\sum_{a=0}^{a_{1}} r_{p}^{\prime}(a)\left(\frac{p-2}{4}-\frac{p-1}{2^{a+1} p}\right)+|\Delta|\left(\frac{p-2}{4 p}\right)-\frac{p-1}{2 p} s .
\end{aligned}
$$

Since $G$ is not a 2-group, so we have either $\sum_{a=0}^{a_{0}} r_{p}^{\prime}(a)>0$ or $s>0$. If some $r_{p}^{\prime}(a)>0$, then

$$
m>\frac{n}{4}+\frac{p-2}{4}-\frac{p-1}{2^{a+1} p}
$$

But we note that since $p \geqslant 5$, for each $a \geqslant 0$,

$$
\frac{p-2}{4}-\frac{p-1}{2^{a+1} p} \geqslant \frac{p-2}{4}-\frac{p-1}{2 p}>0 .
$$


Hence,

$$
m>\frac{n}{4}+\frac{p-2}{4}-\frac{p-1}{2 p}=\frac{n}{4}+\frac{p^{2}-4 p+2}{4 p} .
$$

On the other hand if $s>0$, then $|\Delta| \geqslant(p+1) s \geqslant p+1$. Thus,

$$
\begin{aligned}
m & >\frac{n}{4}+|\Delta|\left(\frac{p-2}{4 p}\right)-\frac{p-1}{2 p} s \\
& \geqslant \frac{n}{4}+s\left(\frac{(p+1)(p-2)}{4 p}-\frac{p-1}{2 p}\right) \\
& \geqslant \frac{n}{4}+\frac{p^{2}-3 p}{4 p} .
\end{aligned}
$$

So in either case we must have,

$$
m>\frac{n}{4}+\min \left\{\frac{p^{2}-4 p+2}{4 p}, \frac{p^{2}-3 p}{4 p}\right\}=\frac{n}{4}+\frac{p^{2}-4 p+2}{4 p} .
$$

Hence,

$$
n<4 m-\frac{p^{2}-4 p+2}{4 p}=4 m-p+4-\frac{2}{p} .
$$

That is, $n<4 m-p+4$ and so $n \leqslant 4 m-p+3$. Hence the proof of Theorem 1.1 is complete.

\section{The PROOF OF TheOREM 1.2}

In this section with the notation defined in Section 3 we refine the upper bound $4 m-p+3$ for $n$ and obtain an upper bound that is attained for an infinite family of groups. For this purpose suppose that $G$ is not a 2 -group on any orbit, that is $u=0$. Then we have the following lemma.

LEMMA 4.1. With the above notation,

$$
n<\frac{2 p^{2} m}{(p-1)^{2}}-\left[\frac{p}{p-1} \sum_{a} \frac{2^{a}-1}{2^{a}} r_{p}^{\prime}(a)+\frac{1}{p-1}|\Delta|-\frac{p}{p-1} s\right] .
$$

Proof: Since $G$ is not a 2-group on any orbit, $u=0$ and so $|\Phi|=0$ the inequality of Lemma 3.2, yields

$$
m>\left(\sum_{a=0}^{a_{0}} \frac{p-1}{2} \cdot\left(1-\frac{1}{2^{a} p}\right) r_{p}^{\prime}(a)+\frac{p-1}{2 p}(|\Delta|-s)\right) .
$$

Since $n=p r_{p}+p r_{p}^{\prime}+|\Delta|$, we have

$$
\begin{aligned}
\frac{2 p^{2} m}{(p-1)^{2}} & >p r_{p}+\frac{p}{p-1} \sum_{a} \frac{2^{a} p-1}{2^{a}} r_{p}^{\prime}(a)+\frac{p}{p-1}(|\Delta|-s) \\
& =p r_{p}+p r_{p}^{\prime}+\frac{p}{p-1} \sum_{a} \frac{2^{a}-1}{2^{a}} r_{p}^{\prime}(a)+|\Delta|+\frac{1}{p-1}|\Delta|-\frac{p}{p-1} s .
\end{aligned}
$$


Hence,

$$
\frac{2 p^{2} m}{(p-1)^{2}}>n+\left[\frac{p}{p-1} \sum_{a} \frac{2^{a}-1}{2^{a}} r_{p}^{\prime}(a)+\frac{1}{p-1}|\Delta|-\frac{p}{p-1} s\right],
$$

and we have the stated inequality.

Now define $\eta:=2 p^{2} m-(p-1)^{2} n$. Clearly $\eta$ is an integer and from Lemma 3.3 we have that

$$
0 \leqslant p(p-1) \sum_{a} \frac{2^{a}-1}{2^{a}} r_{p}^{\prime}(a)+(p-1)|\Delta|-(p-1) p . s<\eta .
$$

If $\eta>p(p-1)$, then part 1 of Theorem 1.2 follows. To prove the theorem we suppose that $\eta \leqslant p(p-1)$ and seek to discover what configurations may occur.

From the inequality

$$
0 \leqslant p(p-1) \sum_{a} \frac{2^{a}-1}{2^{a}} r_{p}^{\prime}(a)+(p-1)|\Delta|-(p-1) p . s<\eta \leqslant p(p-1)
$$

we obtain,

$$
0 \leqslant p \sum_{a} \frac{2^{a}-1}{2^{a}} r_{p}^{\prime}(a)+(|\Delta|-(p-1) p . s)<p .
$$

First we show that $s=0$. Since if $s \neq 0$, then by [4, Lemma 2]

$$
p . t<n \leqslant t+\frac{2 m p}{p-1}-1 \text {. }
$$

Hence,

$$
t<\frac{1}{p-1}\left(\frac{2 m p}{p-1}-1\right)
$$

and then we have

$$
n \leqslant t+\frac{2 m p}{p-1}-1<\frac{p}{p-1}\left(\frac{2 m p}{p-1}-1\right),
$$

which contradicts our assumption. Hence $s=0$, and so $|\Delta|=0$ and

$$
0 \leqslant p \sum_{a} \frac{2^{a}-1}{2^{a}} r_{p}^{\prime}(a)<\eta /(p-1) \leqslant p .
$$

This inequality implies that $r_{p}^{\prime} \leqslant 1$. If $r_{p}=0$ and $r_{p}^{\prime} \neq 0$, then $n=p=2 m p /(p-1)$. Following [2] and Lemma 2.3, the last equality holds if and only if $m=(p-1) / 2$ and $G=Z_{2^{a}} \ltimes Z_{p}$, where $a \geqslant 1$, and $2^{a} \mid(p-1)$.

Moreover, suppose that $r_{p}^{\prime}=0$. Then $n=p . r_{p}$ and by Example $2.2 G$ is an elementary Abelian p-group.

Now we consider the case that $r_{p}>0$, and $r_{p}^{\prime}>0$, that is $r_{p}^{\prime}=1$. Define $\Sigma_{1}$ $:=\bigcup_{i=1}^{r_{p}} \Omega_{i}$, the union of the orbits of length $p$ on which $G$ acts as $Z_{p}$, and $\Sigma_{2}$, the unique of the orbit of length $p$ on which $G$ acts as $G=Z_{2^{a}} \ltimes Z_{p}$, where $a \geqslant 1$ and $2^{a} \mid(p-1)$. 
Let $m_{1}:=\operatorname{move}\left(G^{\Sigma_{1}}\right)$, and $m_{2}:=\operatorname{move}\left(G^{\Sigma_{2}}\right)=(p-1) / 2$. By Example 2.2 each non-identity element of $G^{\Sigma_{1}}$ have the same movement $m_{1}$, so by considering of the each $p$-element of $G^{\Sigma_{2}}$ it follows easily that $m=m_{1}+m_{2}$. Defining $n_{1}:=\left|\Sigma_{1}\right|$, and $n_{2}$ $:=\left|\Sigma_{2}\right|=p=2 m p /(p-1)$. We have from Example 2.2 that

$$
n_{1} \leqslant \frac{p}{p-1}\left(\left\lfloor\frac{2 m_{1} p}{p-1}\right\rfloor-1\right)
$$

and so

$n=n_{1}+n_{2} \leqslant \frac{p}{p-1}\left(\left\lfloor\frac{2 m_{1} p}{p-1}\right\rfloor-1\right)+\left\lfloor\frac{2 m_{2} p}{p-1}\right\rfloor<\frac{p}{p-1}\left(\left\lfloor\frac{2 m_{1} p}{p-1}\right\rfloor-1\right)+\frac{p}{p-1}\left\lfloor\frac{2 m_{2} p}{p-1}\right\rfloor$.

Hence,

$$
n<\frac{p}{p-1}\left(\left\lfloor\frac{2 m p}{p-1}\right\rfloor-1\right)
$$

What this has shown is that, if $\eta:=2 p^{2} m-(p-1)^{2} n$, that is, $n \geqslant(\lfloor(2 m p / p-1)\rfloor$ $-1) /(p-1)$, then either $r_{p}=0$ or $r_{p}^{\prime}=0$ and we have the situation posed in above. In this cases

$$
n=\left\lfloor\frac{2 m p}{p-1}\right\rfloor=\frac{p}{p-1}\left(\left\lfloor\frac{2 m p}{p-1}\right\rfloor-1\right) .
$$

Thus the result now follows and the proof of Theorem 1.2 is complete.

\section{REFERENCES}

[1] J.R. Cho, P.S. Kim, and C.E. Praeger, 'The maxiamal number of orbits of a permutation groups with bounded movement', J. Algebra 214 (1999), 625-630.

[2] A. Hassani, M. Khayaty, E. I. Khukhro and C. E. Praeger, 'Transitive permutation groups with bounded movement having maximal degree', J. Algebra 214 (1999), 317-337.

[3] P.M. Neumann and C.E.Praeger, 'On the movement of a permutation group', J. Algebra 214 (1999), 631-635.

[4] C.E. Praeger, 'On permutation groups with bounded movement', J. Algebra 144 (1991), 436-442.

[5] J.J. Rotman, An Introduction to the theory of groups, (3rd edition) (Allyn and Bacon, Boston, 1984).

[6] T. Tsuzuku, Finite groups and finite geometries (Cambridge University Press, Cambridge, 1982).

Department of Mathematics

Iran University of Science and Technology

Narmak

Tehran 16844

Iran

e-mail: Khayaty@iust.ac.ir 